\title{
Numerical prediction and wind tunnel experiment for a pitching unmanned combat air vehicle
}

\author{
Russell M. Cummings, Scott A. Morton, Stefan G. Siegel \\ Department of Aeronautics, United States Air Force Academy, USAF Academy, CO 80840, USA
}

\begin{abstract}
The low-speed flowfield for a generic unmanned combat air vehicle (UCAV) is investigated both experimentally and numerically. A wind tunnel experiment was conducted with the Boeing 1301 UCAV at a variety of angles of attack up to 70 degrees, both statically and with various frequencies of pitch oscillation $(0.5,1.0$, and $2.0 \mathrm{~Hz})$. In addition, pitching was performed about three longitudinal locations on the configuration (the nose, 35\% MAC, and the tail). Solutions to the unsteady, laminar, compressible Navier-Stokes equations were obtained on an unstructured mesh to match results from the static and dynamic experiments. The computational results are compared with experimental results for both static and pitching cases. Details about the flowfield, including vortex formation and interaction, are shown and discussed, including the non-linear aerodynamic characteristics of the vehicle.
\end{abstract}

Published by Elsevier Masson SAS.

Keywords: Unsteady aerodynamics; Computational fluid dynamics; Wind tunnel testing; UCAV; Maneuvering aircraft

\section{Introduction}

Unmanned combat air vehicles (UCAVs) have shown their value as reconnaissance vehicles, and even tactical weapons, over the past few years. Aircraft such as Predator and Global Hawk are fast becoming essential tools in the day-to-day operations of the military. Eventually, UCAVs will be called upon to take advantage of their pilotless state and pull many more g's than manned aircraft. Issues such as control actuation [12], morphing wings [11], fuel cell-based propulsion systems [2], MEMS-based control systems [18], and semiautonomous flight [3] will be essential to the further development of these vehicles. One such capability will be utilizing dynamic lift (also known as dynamic stall) due to fast pitch-ups for super-maneuverability and agility.

Dynamic lift utilizes hysteresis effects and vortex formation on airfoils or wings pitching up at rapid rates to delay the onset of stall. As airfoils pitch up there is a time lag in the separation of flow over the upper surface, which allows for the attainment of higher angles of attack than during static conditions. In addition, leading-edge vortices form that aid in the development of lift. Several researchers have shown the effects of dynamic stall on airfoils, both with experimental and numerical studies [5, 13,15]. In fact, excellent review articles on dynamic stall have been written by Ekaterinaris and Platzer [9], as well as Carr [4]. Experimental and numerical studies have also been conducted on wings undergoing dynamic stall, including rectangular and swept wing $[6,17,22,24]$ as well as delta wings $[19,23]$. Very little work, however, has been done on studying the dynamic lifting capabilities of full aircraft configurations with mid-range levels $\left(30^{\circ}\right.$ to $\left.60^{\circ}\right)$ of sweep, such as generic UCAVs [16]. This research hopes to better understand the impact of vortex lift and vortex breakdown, coupled with dynamic lift, for these configurations.

The results of dynamic stall studies for airfoils may be summarized by the lift and pitching moment results shown in Fig. 1. Depending on the pitching frequency, an airfoil will exceed 


\section{Nomenclature}

$b \quad$ wing span, 12 in $(0.305 \mathrm{~m})$

$\bar{c} \quad$ mean aerodynamic chord, 5.24 in $(0.133 \mathrm{~m})$

$C_{D} \quad$ drag coefficient, $\equiv D / q_{\infty} S_{\text {ref }}$

$C_{L} \quad$ lift coefficient, $\equiv L / q_{\infty} S_{\text {ref }}$

$C_{m} \quad$ pitching moment coefficient, $\equiv m / q_{\infty} S_{\text {ref }} \bar{c}$

$C_{N} \quad$ normal force coefficient, $\equiv N / q_{\infty} S_{r e f}$

$D \quad$ drag

E modulus of elasticity for wing material

$f \quad$ pitching frequency

$\mathrm{Hz}$

$k \quad$ reduced frequency, $\equiv \pi f \bar{c} / V_{\infty}$

$L \quad$ lift

$l \quad$ vehicle length, 8.50 in $(0.216 \mathrm{~m})$

$m$ pitching moment (about $35 \%$ mean aerodynamic chord)

$\begin{array}{ll}q & \text { dynamic pressure, } \equiv \rho V^{2} / 2 \\ S_{r e f} & \text { wing planform area, } 46.82 \mathrm{in}^{2}\left(302.1 \mathrm{~cm}^{2}\right) \\ s & \text { wing semi-span, } \equiv b / 2 \\ S t & \text { Strouhal number, } \equiv f \bar{c} / V_{\infty} \\ t & \text { physical time or wing thickness } \\ t^{*} & \text { non-dimensional time, } \equiv t V_{\infty} / \bar{c} \\ V & \text { velocity } \\ V_{\infty} & \text { freestream velocity, } 65.4 \mathrm{ft} / \mathrm{s}(20 \mathrm{~m} / \mathrm{s}) \\ x_{c p} & \text { center of pressure, } \equiv-\bar{c} C_{m} / C_{N} \\ \alpha & \text { angle of attack } \ldots \ldots \ldots \ldots \ldots \ldots \text { deg } \\ v & \text { Poisson's ratio for wing material } \\ \rho & \text { air density } \\ \omega & \text { rotation rate, }=2 \pi f \\ \infty & \text { freestream condition }\end{array}$
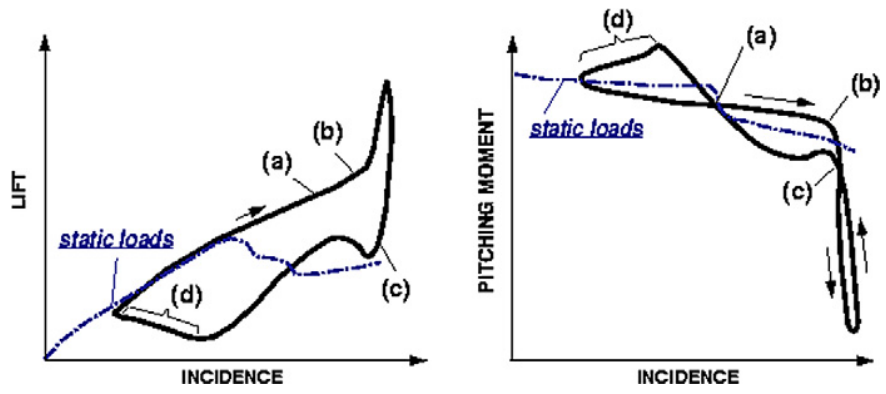

Fig. 1. Dynamic stall events on an airfoil (Refs. [9] and [4]).

static loads as it pitches up, with lift continuing to increase until separation becomes apparent on the upper surface near the trailing edge (a). Eventually, as the separation region grows, a vortex will form near the leading edge of the airfoil (b). The vortex momentarily increases the rate of lift increase, but eventually the stall region reaches its full extent (c). Finally, as the airfoil pitches down the boundary layer begins reattaching to the upper surface, and the flow returns to its original state (d). The combination of flow separation and vortex formation also has a large impact on pitching moment, as can also be seen in Fig. 1. Again, these results are based on twodimensional airfoils pitching at approximately the same frequencies.

Slender delta wings exhibit similar behavior as airfoils, however for very different reasons $[1,16,19,23]$. Delta wings create leading edge vortices, which cause non-linear lifting characteristics. As the angle of attack is increased, however, vortex breakdown causes this lift to decrease, eventually leading to stall. Under unsteady pitching, the vortex breakdown phenomenon experiences a hysteresis which delays stall. Eventually the pitching delta wing stalls (although at a higher angle of attack than in the static case), and as the wing pitches down the forces and moments return to their static values [20]. It is not known whether these results would be reproduced on a full UCAV configuration.

\section{Experimental approach}

A full-scale model for the Boeing 1301 UCAV configuration is shown in Fig. 2; the configuration has many similar features to the X-45A UCAV configuration (see Fig. 3). The 1301 configuration has a straight, $50^{\circ}$ sweep leading edge, an aspect ratio of 3.1, a top-mounted engine inlet, and a B-2-like wing planform. The full-scale configuration has a mean aerodynamic chord of $20.2 \mathrm{ft}$ and a reference area of $694 \mathrm{ft}^{2}$.

A 1:46.2 scale model of the configuration was tested in the USAF Academy $3 \mathrm{ft} \times 3 \mathrm{ft}(0.914 \mathrm{~m} \times 0.914 \mathrm{~m})$ open return low-speed wind tunnel (see Fig. 4). The scaled model has a mean aerodynamic chord of 5.24 in $(0.133 \mathrm{~m})$ and a reference area (wing planform area) of $46.82 \mathrm{in}^{2}\left(302.1 \mathrm{~cm}^{2}\right)$. The tunnel has less than $0.05 \%$ freestream turbulence levels at all speeds. The test was conducted at a freestream velocity of $65.4 \mathrm{ft} / \mathrm{s}$ ( $20 \mathrm{~m} / \mathrm{s})$, which corresponds to a chord-based Reynolds number of $1.42 \times 10^{5}$. The model was sting-mounted from the rear, and forces and moments were measured with a six-component force balance, with a normal force range of $50 \mathrm{lb}(223 \mathrm{~N})$. Both static and dynamic testing was done; forces during the dynamic runs were obtained by subtracting the force history with the tunnel off from the dynamic data. The balance calibration was accurate to $\pm 0.5 \%$ of the full measurement force of the balance, or $0.25 \mathrm{lb}(1.12 \mathrm{~N})$. It should be noted that the maximum forces measured on the balance were only $15 \%$ to $20 \%$ of the full range of $50 \mathrm{lb}$, which could further add error to the experimental data. The lift and drag coefficients are only accurate to $\pm 1.9 \%$ partially due to inaccurate readings of the room static pressures on the testing days.

The dynamic pitching was done with a shifted cosine oscillation, starting at a certain angle of attack and pitching up to twice the peak amplitude of the cosine wave, then back to the original angle of attack. The pitch variation was defined as:

$\alpha(t)=\alpha_{\circ}+m^{\circ}(1-\cos (\omega t))$

where $\alpha_{\circ}$ and $m$ were varied to obtain results for $0^{\circ} \leqslant \alpha \leqslant 45^{\circ}$ in three pitching cycles. This pitching function was used since 

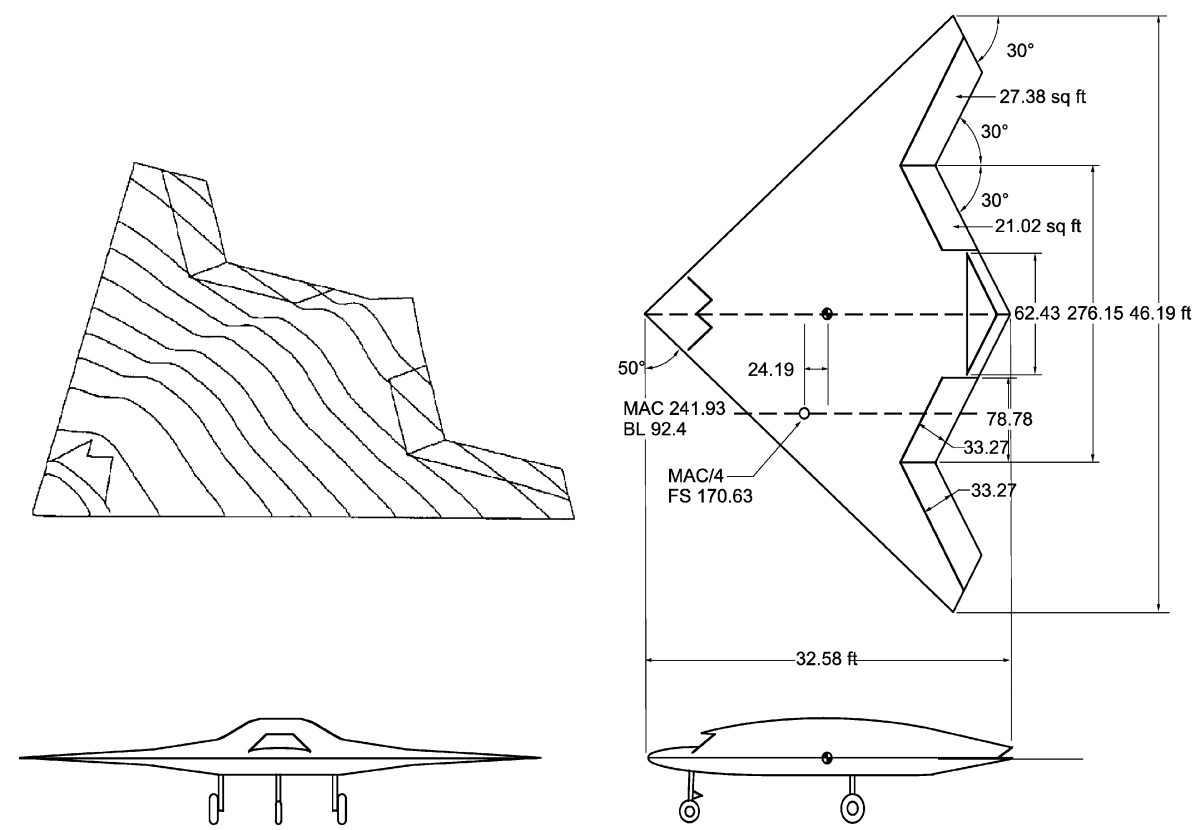

Fig. 2. Boeing 1301 UCAV configuration.

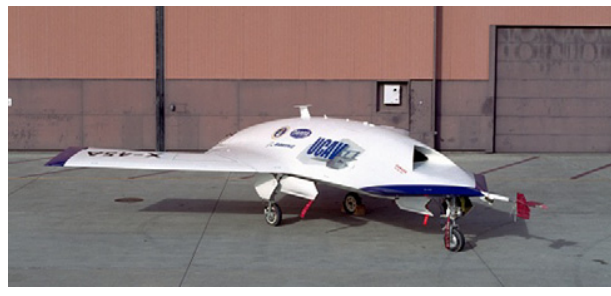

Fig. 3. X-45A UCAV configuration (photo courtesy of NASA Dryden Flight Research Center).

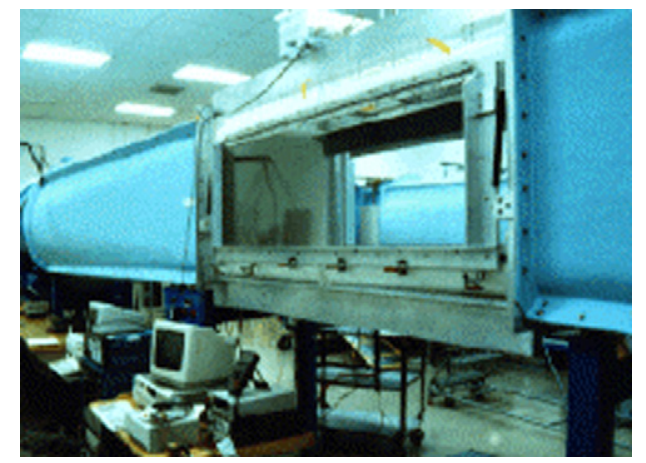

Fig. 4. USAF Academy $3 \mathrm{ft} \times 3 \mathrm{ft}$ low-speed wind tunnel.

it produces a motion without any discontinuities in acceleration or velocity at the beginning and end of the motion, thus being easier to implement in an experiment or a CFD code.

The model was suspended from downstream using a $\mathrm{C}$-shaped bracket with a center mount for the balance and model. The bracket was mounted vertically in the test section of the tunnel, so that the center of rotation was a vertical axis through the center of the tunnel. Two separate synchronized servo motors on the top and bottom of the tunnel drove the pitching motion through a timing belt/arc sector with a $20: 1$ gear ratio setup.

\section{Experimental results}

The configuration was first tested in a static mode for angles of attack ranging from $0^{\circ} \leqslant \alpha \leqslant 70^{\circ}$; the resulting lift and drag coefficients, as well as the center of pressure, are presented in Fig. 5. As can be seen, the configuration has linear lift characteristics up to an angle of attack of $\alpha=10-12^{\circ}$, with stall occurring at approximately $\alpha=20^{\circ}$. The stall is not deep, however, and the configuration quickly re-establishes an increasing lift variation up to $\alpha=32^{\circ}$, where an abrupt loss of lift takes place. The cause of the increased lift above the initial stall angle of attack, and the loss of lift above $\alpha=32^{\circ}$ were not initially known, but will be explained in a later section. The configuration is unstable (as most flying wing configurations are) with the center of gravity at 35\% MAC with pitch reversal at $\alpha=20^{\circ}$, $32^{\circ}$, and $46^{\circ}$. The neutral point for the configuration at low speeds and low angles of attack is approximately $20 \%$ MAC, which means that the aircraft will be slightly unstable for most realistic center of gravity locations.

The configuration was then pitched at $0.5,1.0$, and $2.0 \mathrm{~Hz}$ ( $k=0.01,0.02$, and 0.04 , respectively), with the center of rotation being at the nose, $35 \%$ of mean aerodynamic chord (MAC), and the tail. The pitch cycles were completed for three ranges of angle of attack: $0^{\circ} \leqslant \alpha \leqslant 20^{\circ}, 16^{\circ} \leqslant \alpha \leqslant 35^{\circ}$, and $25^{\circ} \leqslant \alpha \leqslant 45^{\circ}$. A representative set of experimental data is presented in Fig. 6, where the pitch rate is $2 \mathrm{~Hz}(k=0.04)$ and the pitching is about the 35\% MAC location (a nominal c.g. location). The static lift and drag results are also presented for reference.

Notice the hysteresis for $0^{\circ} \leqslant \alpha \leqslant 20^{\circ}$, with increased lift (relative to the static case) being obtained during the pitch-up motion through the past-stall region. During the pitch-down motion there is decreased lift for the remainder of the cycle. In addition, the hysteresis effect decreases as the angle of attack range increases. Also, while the pitching effect on lift is 

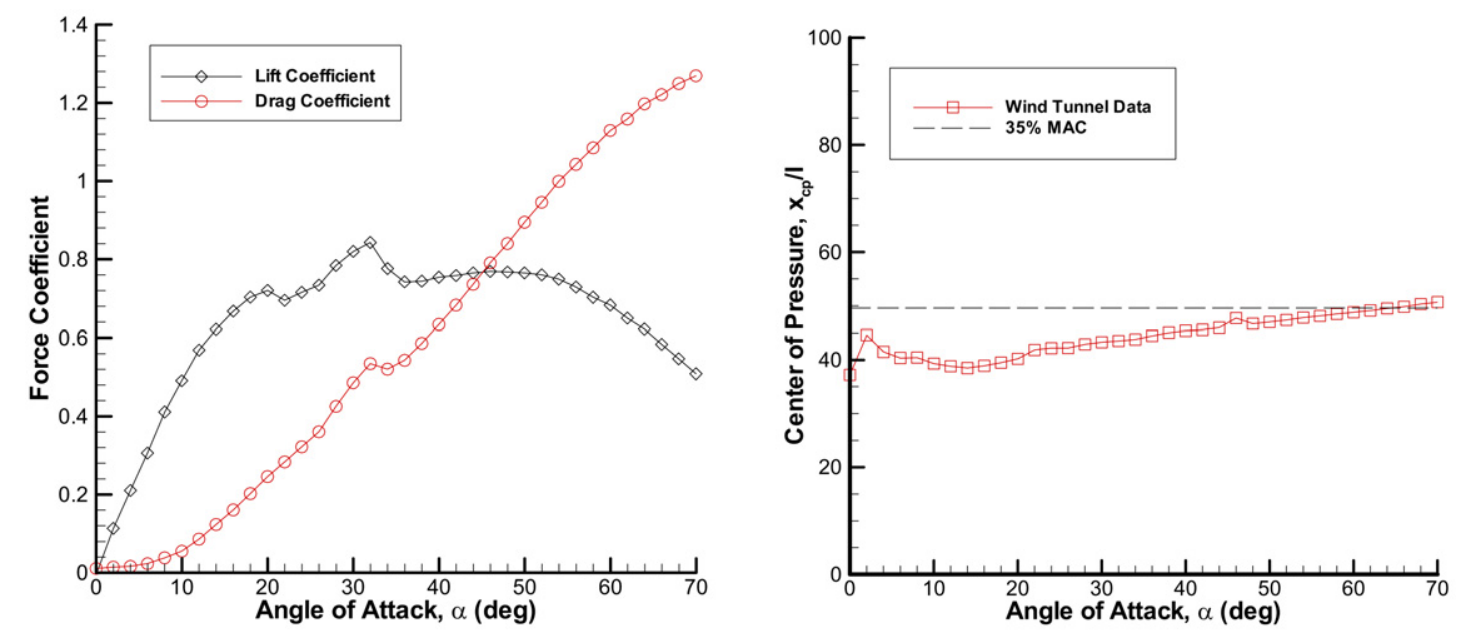

Fig. 5. Experimental longitudinal static force coefficients and center of pressure for the UCAV 1301 configuration.

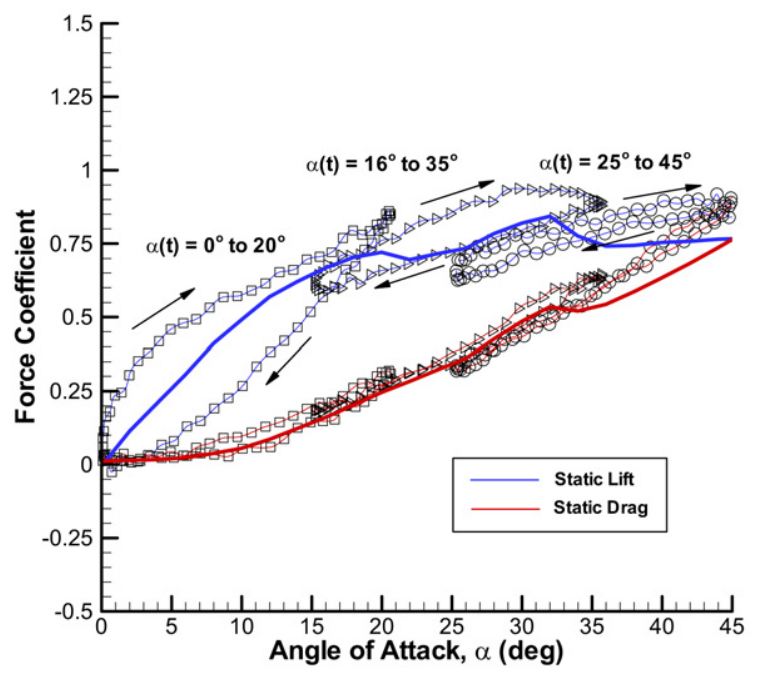

Fig. 6. Experimental pitch-up forces (pitching about 35\% MAC at $2 \mathrm{~Hz}$, $k=0.04$ ).

obvious, there is very little impact on the drag of the configuration. The results are not similar to airfoil or delta wing results, since airfoils and delta wings rarely gain or lose lift at angles of attack in the linear range during the pitch-up or pitch-down motion. While these results are interesting, very little knowledge is gained about the fluid dynamic processes that cause the experimental results due to the integrated affect of measuring forces.

Other representative results for pitching about the nose and tail are presented in Figs. 7 and 8, respectively (Figs. 6-8 all use the same scales for comparison purposes). While these pitching centers are not normally feasible for conventional configurations, they could be attained with vectored thrust or pneumatic flow control. Notice that each of these cases has somewhat different characteristics than the $35 \%$ MAC case, with pitching about the tail providing the biggest differences when compared with the other cases.

While the results for pitching about the nose (Fig. 7) only show slight differences when compared with the 35\% MAC center of pitch results, pitching about the tail (Fig. 8) shows

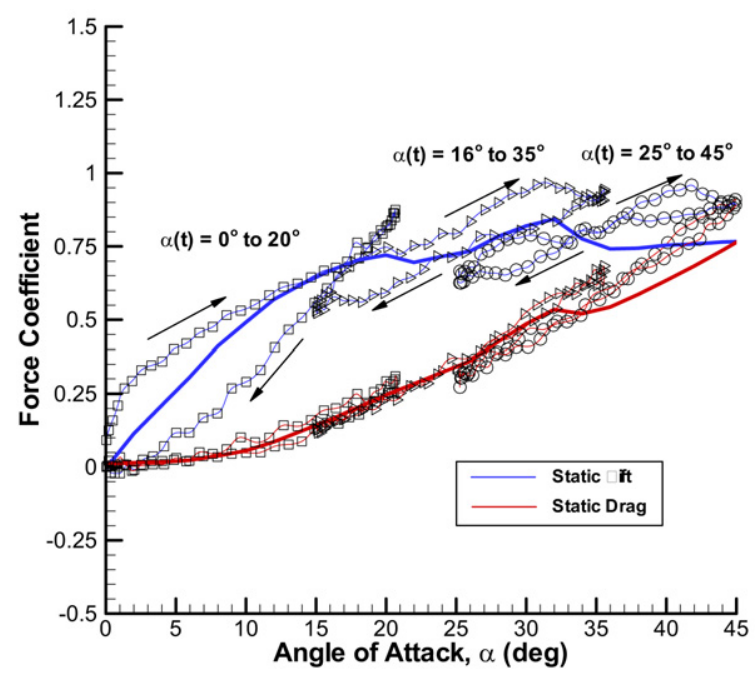

Fig. 7. Experimental pitch-up forces (pitching about the nose at $2 \mathrm{~Hz}, k=0.04$ ).

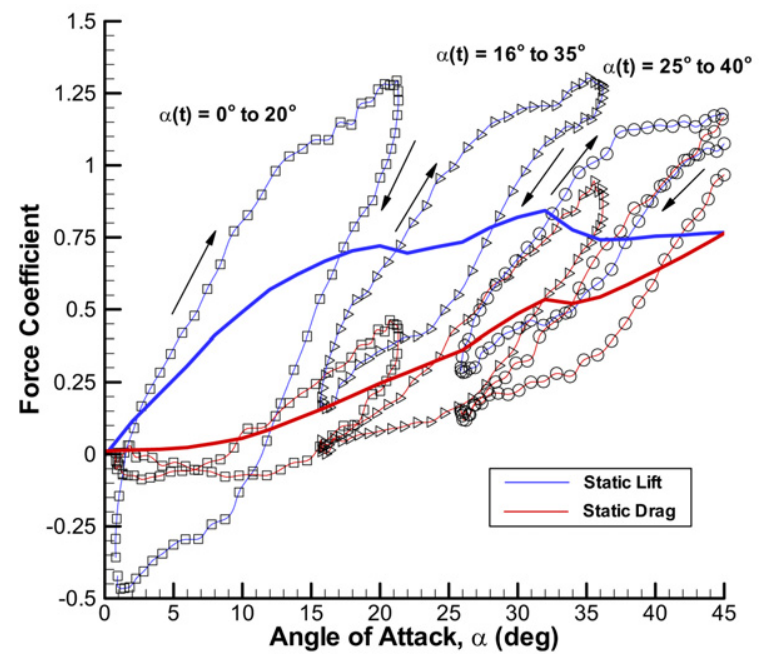

Fig. 8. Experimental pitch-up forces (pitching about the tail at $2 \mathrm{~Hz}, k=0.04$ ). 
markedly different results. While the pitch-up portion of the cycle yields dramatically higher lift coefficients in the post-stall region, the pitch-down lift is dramatically lower than the static results for most of the cycle. Pitching about the tail, while interesting, may not afford the overall increases in lift that would be of interest in a UCAV, with the lift increase during the pitch-up being counterbalanced by the lift decrease during the pitchdown, except in the post-stall region. This might lead to the use of various flow control methods to obtain similar results without the adverse impact on lift at the lower angles of attack.

\section{Numerical approach}

The unstructured flow solver Cobalt (a commercial version of Cobalt 60 ) was chosen because of its speed and accuracy, as well as our experience in using it for massively separated flowfields. Cobalt solves the Euler or Navier-Stokes equations, including an improved spatial operator and improved temporal integration. The code has been validated on a number of problems utilizing turbulence models, including the SpalartAllmaras model [25]. Tomaro, et al., converted Cobalt 60 from explicit to implicit, enabling CFL numbers as high as one million [27]. Grismer, et al., then parallelized the code, yielding a linear speedup on as many as 1024 processors [14]. Forsythe, et al., provided a comprehensive testing and validation of the RANS models, including the Spalart-Allmaras, Wilcox $k-\omega$, and Menter's turbulence models [10].

The computations were performed on an Origin 2000 computer (using 30 processors) and a Beouwulf cluster (using 38 processors). The solutions were obtained using unstructured grids with a combination of prisms and tetrahedrons. The meshes were generated for half of the configuration, with symmetry assumed about the configuration centerline in the spanwise direction. This was deemed acceptable for high angle of attack flow predictions because the left and right vortices have very little interaction due to the span of the UCAV configuration, and very little flow asymmetry was expected. The centerline plane was modeled as a symmetry plane, the UCAV surface was modeled as a solid wall with a no slip condition, and the outer boundaries were modeled with freestream conditions. In order to more closely match the wind tunnel model, the inlet and exhaust areas of the configuration were covered over with solid surfaces. The outer boundary was placed 8 mean aerodynamic chords in front of, 10 mean aerodynamic chords behind, and 4.5 mean aerodynamic chords above and below the configuration.

The flowfield for the 1301 UCAV configuration was computed for three grids of varying sizes: 1.3 million, 2 million, and 4 million cells. The 2 million cell mesh is shown in Fig. 9. Mesh refinement was made in the region above the wing in order to more effectively model the leading-edge vortices and vortex breakdown above the wing.

A grid resolution study was performed with the three grids by running each grid to a steady-state solution at $\alpha=20^{\circ}$. The normal force variation with iterations is shown in Fig. 10 for the coarse, medium, and fine grids, 1.3, 2.0, and 4.0 million cells, respectively. While both solutions show that the results

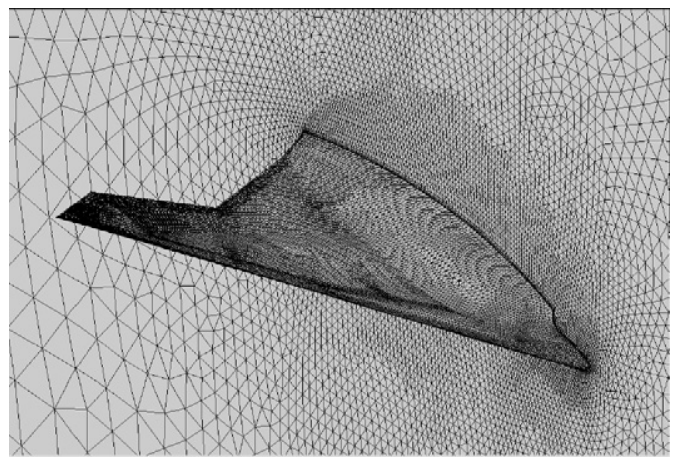

Fig. 9. UCAV 1301 with 2 million cell mesh.

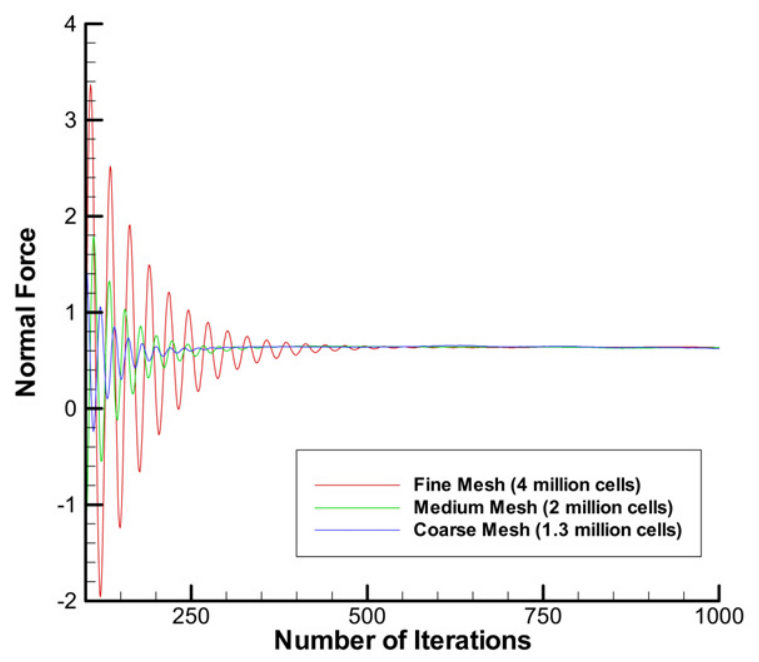

Fig. 10. Grid resolution study at $\alpha=20^{\circ}$.

are somewhat unsteady, they have converged to the same normal force levels. Based on these results, all further computations have been performed on the 2.0 million cell mesh.

\section{Numerical results}

Fig. 11 shows a representative numerical simulation of the configuration at $\alpha=20^{\circ}$, with the flowfield being visualized with streamlines and crossflow planes of $x$-vorticity. The leading-edge vortices are clearly visible closely following the $50^{\circ}$ sweep leading edge, until approximately $x / l=0.60$ when

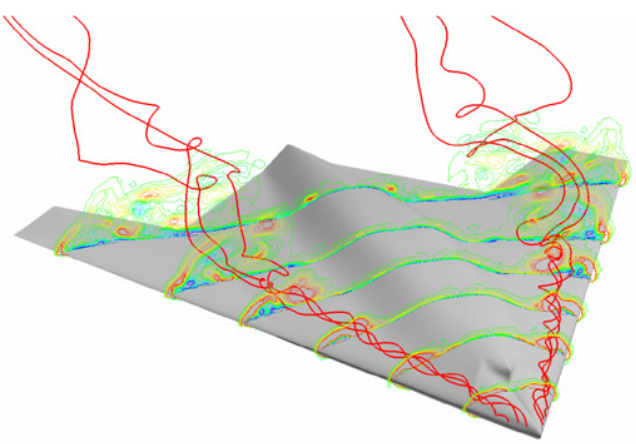

Fig. 11. Steady laminar numerical simulation (crossflow planes of $x$-vorticity with streamlines); $\alpha=20^{\circ}, \operatorname{Re}_{\bar{c}}=1.42 \times 10^{5}$ 

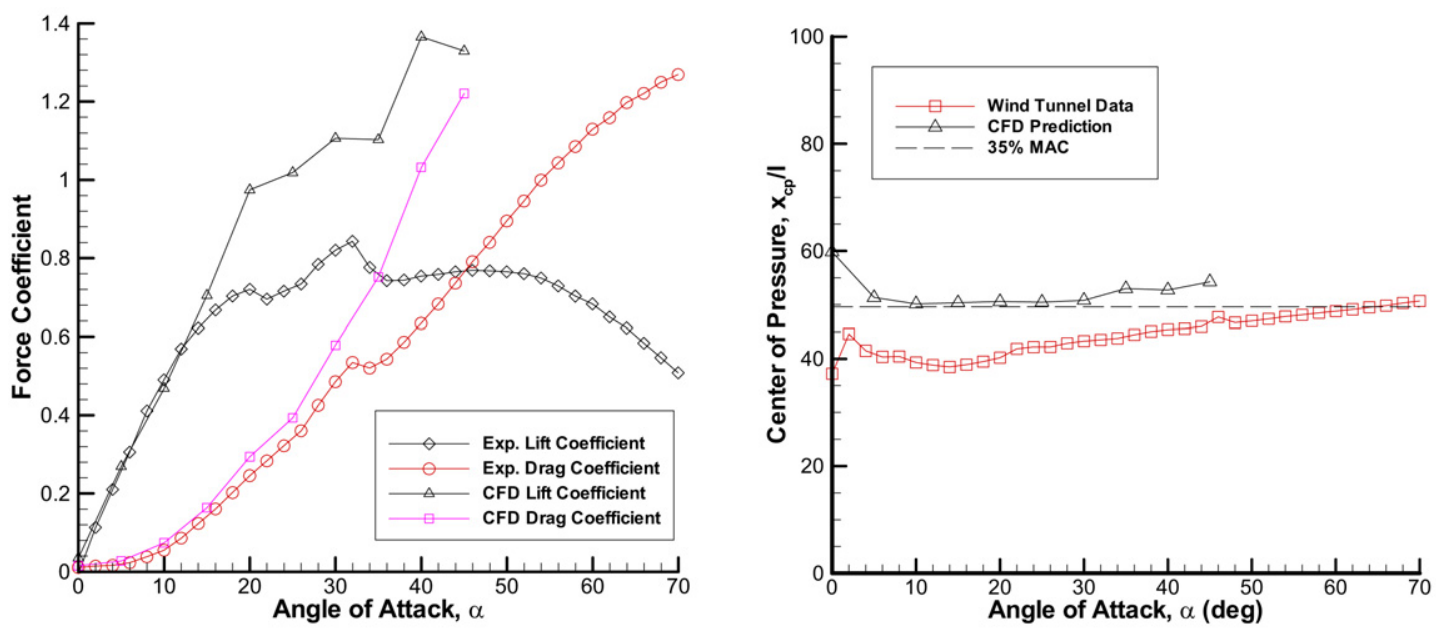

Fig. 12. Numerical (steady) and experimental (static) force coefficient and center of pressure comparisons.

vortex breakdown is evident. Low surface pressures are visible beneath the vortex prior to breakdown; these low pressures account for the lift on the configuration at $\alpha=20^{\circ}$. After breakdown, the vortex wake quickly moves up and behind the leading-edge, leading to higher pressures on the upper surface of the wing. The vortices are very wide compared with their height, most likely due to the rounded leading edges of the wing, and the possible formation of a double primary vortex system $[8,21]$. Secondary vortices are also visible beneath the primary vortices. The primary vortex is seen splitting into two vortical flow structures after the breakdown location.

Fig. 12 shows a comparison between steady numerical results (first-order accurate in time) and the static experimental data. The results are quite good for the linear range of angle of attack, but do not capture the stall region, with the numerical prediction showing the flow remaining attached to a much higher angle of attack. Ordinarily, a fully laminar flow should separate sooner than a turbulent flow, so these results are most likely due to something other than transitional wind tunnel data, such as unsteadiness. The post-stall region is also not predicted well, although general trends seem to mimic the experimental data. Both the magnitudes for lift and drag coefficients in the post-stall region are significantly in error when compared with the data. The center of pressure results show that the CFD prediction is showing the center of pressure in a nominally stable position (compared with a nominal center of gravity located at 35\% MAC), but somewhat aft of the location determined in the wind tunnel tests. This shows the difficult in predicting pitching moments for complex configurations such as the 1301 UCAV.

The numerical predictions for $15^{\circ} \leqslant \alpha \leqslant 45^{\circ}$ were recomputed in time-accurate mode (second-order accurate in time with two Newton sub-iterations per time step, $\Delta t=0.00005 \mathrm{~s}$, $\left.\Delta t^{*}=0.0075\right)$. The post-stall results were fairly dramatically changed after the time-accurate runs (some of which took a considerable amount of time to reach a final solution), with much improved comparisons between the predictions and the experimental data (see Fig. 13). While we would normally expect that a steady solution would give a prediction at the average value of an unsteady solution, this may not always be true when dealing with highly non-linear flowfields as are seen here.

A frequency analysis of the impact of flow unsteadiness on the normal force at $\alpha=20^{\circ}$ showed a dominant frequency with a Strouhal number of $S t=0.23$. This frequency corresponds to the oscillation of the vortex breakdown location, a common unsteady flow feature [16]. A second frequency peak was found for a Strouhal number of $S t=0.82$ (although with an order of magnitude less power than the vortex breakdown frequency), which corresponds to the oscillations caused by vortex shedding from the leading edge. A more detailed study of the causes and locations of unsteadiness would be warranted in future research.

One possible explanation for the poorer comparisons in the stall region is aeroelastic effects. Taylor, et al., [26] showed that nonslender delta wings with flexible structures could display a double stall (or delayed stall or enhanced lift) behavior similar to that seen from the experimental data in Fig. 13. Their wind tunnel results showed that the behavior was especially pronounced on delta wings with between 40 and 55 degrees of leading edge sweep (Fig. 14 shows results for a delta wing with 50 degrees of sweep), but did not occur for delta wings with 60 degrees of sweep or larger. The delayed stall was found to occur due to an anti-symmetric vibration on the wing, where "the effect of flexibility is to reduce the extent to the region of flow reversal that occurs over the wing surface, and to promote the reattachment of the shear layer to higher incidences" [26]. The amount of flexibility required for the delayed stall characteristic to be exhibited was determined by the reciprocal of the spanwise bending stiffness of the wing normalized by the freestream dynamic pressure, $\lambda_{s}$, given by [26]:

$\lambda_{s}=12\left(1-v^{2}\right) \rho_{\infty} V_{\infty}^{2} s^{3} / E t^{3}$

where $v$ is Poisson's ratio for the wing material, $s$ is the wing semi-span, $E$ is the modulus of elasticity for the wing material, and $t$ is the wing thickness. A higher value of $\lambda_{s}$ corresponds to a more flexible wing, and the experiments compared models with flexibility of $\lambda_{s}=3.1$ (highly flexible) and $\lambda_{s}=0.025$ (rigid). For the materials used to make the UCAV 


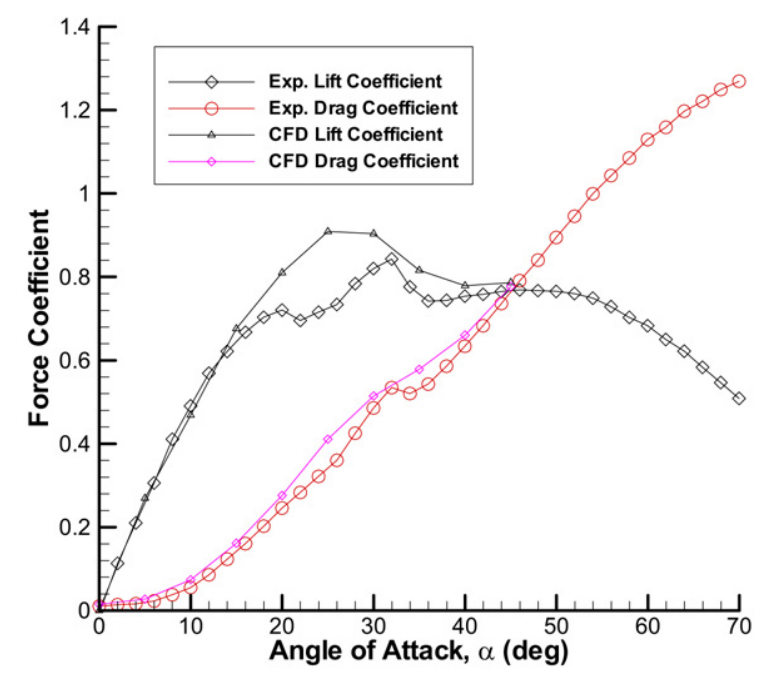

Fig. 13. Numerical (time accurate) and experimental (static) force coefficient comparison.

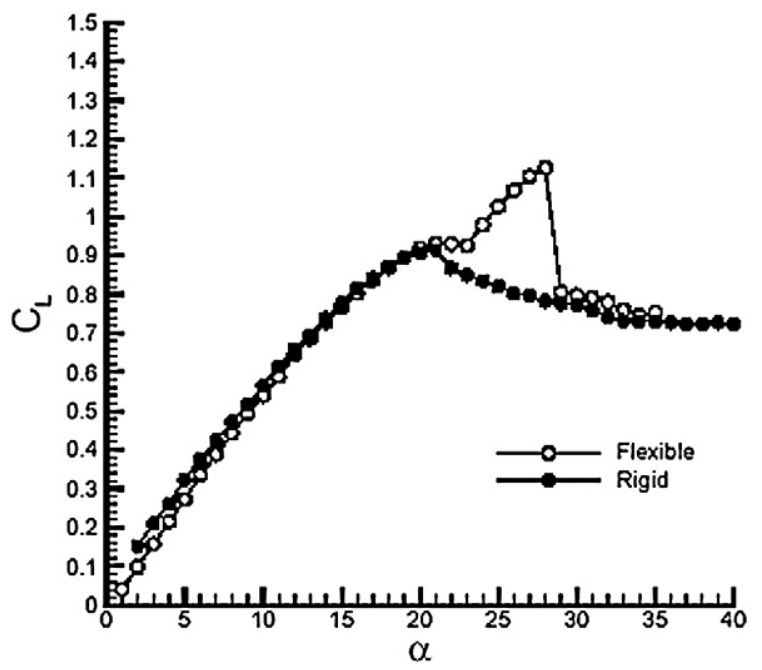

Fig. 14. Stall behavior of rigid and flexible nonslender delta wings with $50^{\circ}$ swept leading edges (from Ref. [26]).

model that was tested in the USAF Academy wind tunnel, $\lambda_{s} \approx 1$, which would be flexible according to the predictions of Ref. [26] (a fact that has been verified by an inspection of the wind tunnel model). Since the CFD predictions are for a wing with no flexibility, aeroelastic effects could possibly account for the difference between the computations and the experimental data seen in Fig. 13.

One of the purposes of the numerical simulation is to visualize the flowfield around the UCAV configuration at various angles of attack and determine what is causing the results seen in Fig. 13. A series of upper surface flowfield visualizations have been made at various angles of attack and are presented in Figs. 15a-15h; all figures use the same pressure scales for comparative purposes. At the lower angles of attack, such as $\alpha=5^{\circ}$ (Fig. 15a), the flow is fairly conventional, with streamlines flowing over the airfoil sections creating lower pressures on the upper surface of the wing. A small region of separated flow is seen near the aft end of the configuration, however.
When the angle of attack is increased to $\alpha=10^{\circ}$ (Fig. 15b), most of the flowfield remains approximately the same, however a small vortex has developed along the leading-edge of the configuration. By the time $\alpha=15^{\circ}$ is reached (Fig. 15c), the leading-edge vortex is quite pronounced, although the vortex is already breaking down approximately half way along the leading edge. While this leading-edge vortex provides additional lift (note the decreased upper surface pressures), the additional lift is not enough to maintain a linear lift curve (as seen in Fig. 13).

At $\alpha=20^{\circ}$ the leading-edge vortex breakdown location has moved forward noticeably, although the vortical flowfield is still maintaining low pressures on the upper surface. As the angle of attack is further increased to $\alpha=25^{\circ}$, the vortex breakdown location has moved forward significantly, with breakdown taking place at approximately $x / l=0.24$ and the leading-edge vortex is only impacting the forward section of the vehicle. In spite of this, the vortex is still producing considerable amounts of lift - the vortex can also be seen to have shifted toward the vehicle centerline, most likely due to the rounded leading edges on the vehicle. Rounded leading edges do not produce nearly as strong a vortex as sharp leading edges, and that impact is certainly affecting the lift of the configuration at these angles of attack. The low pressure region near the nose of the vehicle has become more pronounced, while the pressures over the aft portion of the configuration have increased when compared with the $\alpha=20^{\circ}$ case.

Finally, by the time the vehicle reaches $\alpha=30^{\circ}$ and above, the burst vortex slowly gives way to a largely stalled upper surface, although the leading-edge is still producing a vortex that is creating lift near the front of the vehicle. This explains the fairly constant lift variation with angle of attack from $32^{\circ} \leqslant \alpha \leqslant 50^{\circ}$ (see Fig. 13 and compare with Figs. 15f-15h).

The 1301 UCAV configuration was then run in a dynamic pitch-up to match the pitching motion of the wind tunnel test (see the pitch-up equation previously defined in Eq. (1)). The case was run for the $2 \mathrm{~Hz}$ pitching motion with pitching taking place about the $35 \%$ MAC location. The computations were run at a time step of $\Delta t=0.00005 \mathrm{~s}$, or a non-dimensional time step of $\Delta t^{*}=0.0075$, with 5 Newton sub-iterations. The pitching was initiated from $\alpha=0^{\circ}$ after running for 500 iterations and achieving a steady starting solution.

Fig. 16 shows the pitching results from the computational simulation compared with the wind tunnel data. The pitching cycle begins by transitioning from a steady flowfield and then approaches the pitch-up results from the wind tunnel test. One issue in the results is why the pitching characteristics are different from typical airfoil or delta wing dynamic stall results, which normally pitch up along or near the static lift curve and then extend into the post-stall region (see Fig. 1). After reaching the maximum pitch angle, the lift usually drops below the static value for the remainder of the cycle. In the case of the 1301 UCAV, however, the computed lift is greater than the static case during the full cycle.

Figs. 17 through 19 help explain the results seen in Fig. 15. Fig. 17 shows the UCAV upper surface pressure distribution for the time-accurate static case at $\alpha=15^{\circ}$. Figs. 18 and 19 present the same views for $\alpha \quad 15^{\circ}$ from the pitch-up and pitch-down 


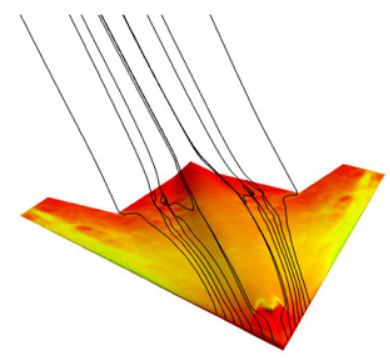

a) $\alpha=5^{\circ}$

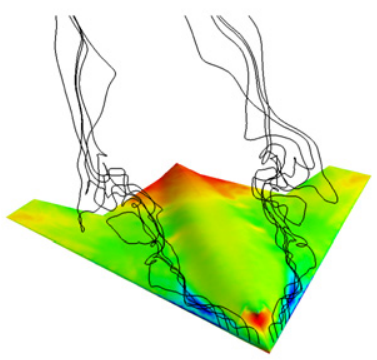

d) $\alpha=20^{\circ}$

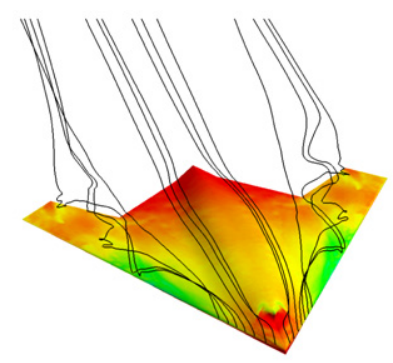

b) $\alpha=10^{\circ}$

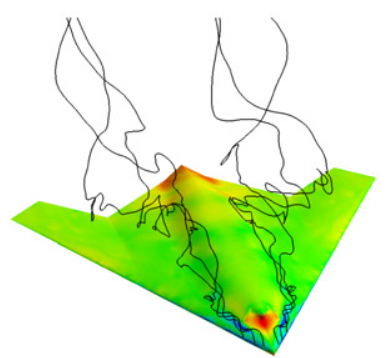

e) $\alpha=25^{\circ}$

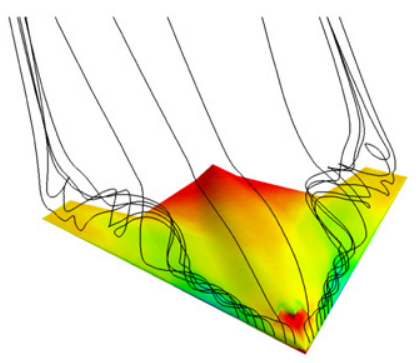

c) $\alpha=15^{\circ}$

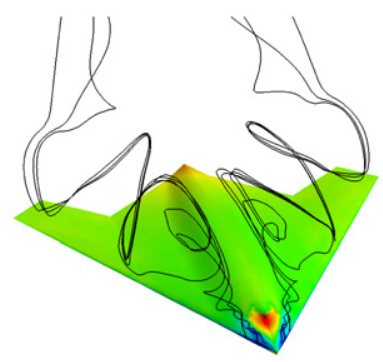

f) $\alpha=30^{\circ}$

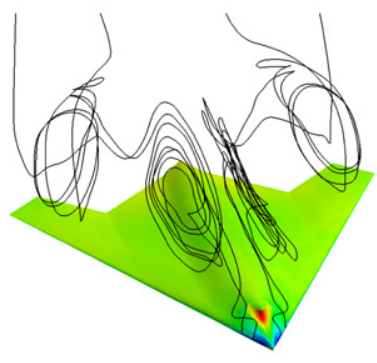

g) $\alpha=35^{\circ}$

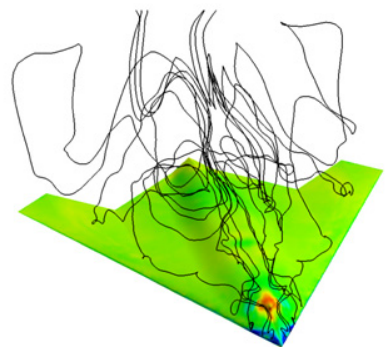

h) $\alpha=40^{\circ}$

Fig. 15. Upper surface flowfields (surfaces colored by pressure; blue $=$ lower pressure, red $=$ higher pressure). (For interpretation of the references to color in this figure legend, the reader is referred to the web version of this article.)

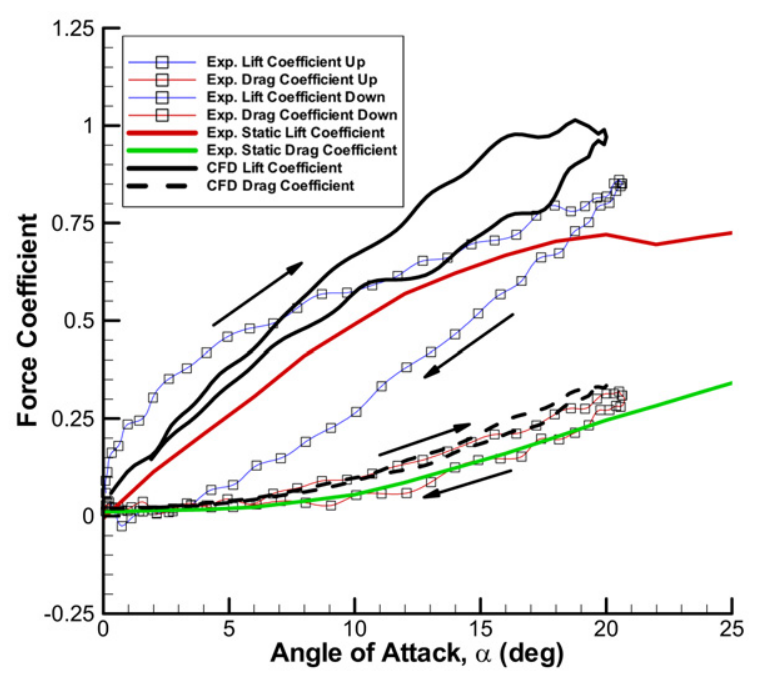

Fig. 16. Pitching cycle comparison; $2 \mathrm{~Hz}(k=0.04)$ pitching about $35 \% \mathrm{MAC}$.

results, respectively. Notice that the leading-edge vortex for the pitch-up case is stronger (resulting in lower pressures), than the static result. This is the cause of the increase in lift during the

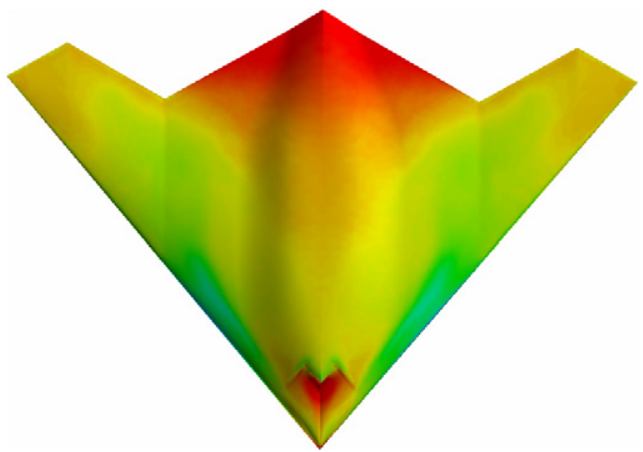

Fig. 17. Static pressure distribution, $\alpha=15^{\circ}$.

pitch-up: the dynamic motion creates a leading-edge vortex that is stronger than for the static case. While the aft portions of the upper surface seem to have slightly different pressures as well, it is believed that most of the dynamic lift is coming from the leading-edge vortex. Since the pitch-up vortex is stronger, vortex breakdown could also take place at lower angles of attack, so there is a trade-off between increased lift during pitch-up and loss of the lift as the vortex breakdown location moves forward 


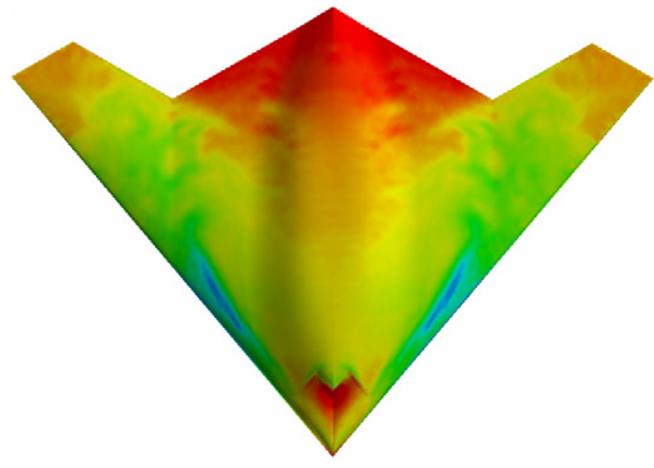

Fig. 18. Pitch-up pressure distribution, $\alpha=15^{\circ} ; 2 \mathrm{~Hz}$ pitching about $35 \%$ MAC.

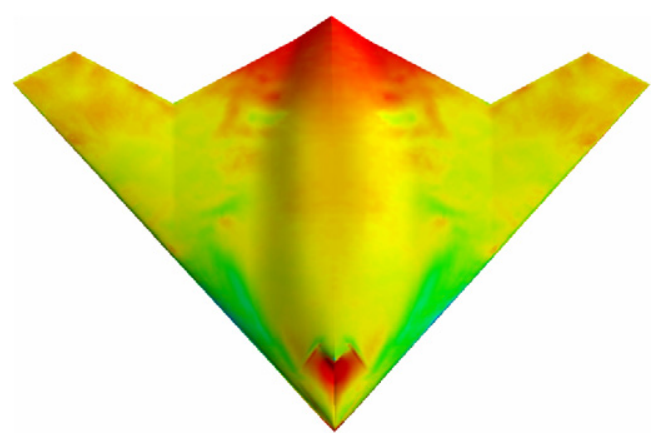

Fig. 19. Pitch-down pressure distribution, $\alpha=15^{\circ} ; 2 \mathrm{~Hz}$ pitching about $35 \%$ MAC.

on the vehicle. This may explain the change in the incremental lift produced while pitching up (see Fig. 15 where the difference between the pitch-up lift and the static lift is more different at lower angles of attack than at higher angles of attack).

Finally, a word should be said about the difficulty in making consistent measurements of unsteady flowfields such as the pitching UCAV shown here. The UCAV geometry displays different characteristics depending on whether the vehicle is pitching or static (as seen in the preceding figures). A question could be asked as to how accurate or repeatable wind tunnel measurements of these flowfields really can be, even if all modern testing techniques are used with high levels of care. Wernert, et al. [28] showed that airfoils undergoing dynamic pitching through stall conditions did not yield repeatable flowfields. They used PIV measurements to quantify the unsteadiness of the wake of the airfoil and found that measurements could not be repeated to desirable levels from one test to another. While this should not keep researchers from performing wind tunnel tests, it does point to the benefits of conducting integrated experimental/computational research [7], so that the strengths of each approach can be fortified and the weaknesses of each approach can be reduced.

\section{Conclusions}

A representative unmanned combat aerial vehicle (UCAV) has been studied computationally and experimentally. The Boeing $1301 \mathrm{UCAV}$ configuration, similar in shape to the $\mathrm{X}-45 \mathrm{~A}$, is a candidate configuration for future UCAV applications, where increased maneuverability and flight capabilities will be important. In order to assess the capabilities of such a configuration, the high angle of attack and pitching characteristics of the vehicle have been assessed.

While the 1301 UCAV is not, in general, an optimum aerodynamic configuration, it does have interesting aerodynamic characteristics. For example, in spite of the use of a rounded leading edge, a leading-edge vortex is clearly developing at $\alpha=10^{\circ}$, and the vortex enables the configuration to continue developing lift up to $\alpha=25^{\circ}$ and beyond. In the post stall region, the vehicle continues to maintain lift, in spite of the fact that the leading-edge vortex has clearly broken down.

The pitching cycle characteristics of the vehicle are somewhat unusual when compared with pitching airfoils and delta wing geometries. Instead of having largely the same lift during a pitch-up maneuver (when compared with the static lift characteristics), the experimental 1301 UCAV results actually gain lift during the pitch-up cycle and lose lift during the pitch-down cycle. The computational results show a lift enhancement during the entire cycle, with the difference probably being caused by aeroelastic effects on the wind tunnel model.

Finally, some recommendations for further study are in order. The UCAV configuration studied here offered a great deal of complexity, and while we have spent a lot of time looking at the configuration, there is certainly more that could be time. The unsteady nature of the flowfield about the 1301 UCAV offers a potential for greater understanding, possibly by investigating the levels of unsteadiness found in local regions of the flow. This would help to determine the causes of the overall unsteady behavior of the vehicle, and would aid in the design of future vehicles. Also, the dynamic lift characteristics of the 1301 UCAV call for more detailed study, especially to determine why this configuration behaves as it seems to.

\section{Acknowledgements}

The authors wish to thank James R. Forsythe of Cobalt Solutions, LLC for his help with using the Cobalt flow solver. We also thank Boeing Military Aircraft in St. Louis, MO for providing the UCAV 1301 geometry.

\section{References}

[1] H. Ashley, J. Katz, M.A. Jarrah, T. Vaneck, Survey of research on unsteady aerodynamic loading of delta wings, Journal of Fluids and Structures 5 (1991) 363-390.

[2] Boeing to develop UAVs with advanced propulsion, Fuel Cells Bulletin (Nov. 2002) 4-5.

[3] B.J. Capozzi, J. Vagners, Evolving (semi)-autonomous vehicles, AIAA Paper 2001-4241, Aug. 2001.

[4] L.W. Carr, Progress in analysis and prediction of dynamic stall, Journal of Aircraft 25 (1) (1988) 6-17.

[5] L.W. Carr, M.S. Chandrasekhara, M.C. Wilder, Effect of compressibility on suppression of dynamic stall using a slotted airfoil, Journal of Aircraft 38 (2) (2001) 296-309.

[6] F.N. Coton, R.A. Galbraith, An experimental study of dynamic stall on a finite wing, Aeronautical Journal 103 (May 1999) 229-236.

[7] R.M. Cummings, S.A. Morton, Continuing evolution of aerodynamic concept development using collaborative numerical and experimental evaluations, Journal of Aerospace Engineering 220 (6) (2006) 545-557. 
[8] R.M. Cummings, S.A. Morton, S.A. Siegel, S. Bosscher, Numerical prediction and wind tunnel experiment for a pitching unmanned combat air vehicle, AIAA Paper 2003-417, Jan. 2003.

[9] J.A. Ekaterinaris, M.F. Platzer, Computational prediction of airfoil dynamic stall, Progress in Aerospace Sciences 33 (1997) 759-846.

[10] J.R. Forsythe, W. Strang, K.A. Hoffmann, Validation of several Reynoldsaveraged turbulence models in a 3D unstructured grid code, AIAA Paper 2000-2552, June 2000.

[11] F.H. Gern, D.J. Inman, R.K. Kapania, Structural and aeroelastic modeling of general planform UCAV wings with morphing airfoils, AIAA Paper 2001-1369, Apr. 2001.

[12] F.H. Gern, D.J. Inman, R.K. Kapania, Computation of actuation power requirements for smart wings with morphing airfoils, AIAA Paper 20021629, Apr. 2002.

[13] D. Greenblatt, D. Neuberger, I. Wygnanski, Dynamic stall control by intermittent periodic excitation, Journal of Aircraft 38 (1) (2001) 188-190.

[14] M.J. Grismer, W.Z. Strang, R.F. Tomaro, F.C. Witzemman, Cobalt: A parallel, implicit, unstructured Euler/Navier-Stokes solver, Advances in Engineering Software 29 (3-6) (1998) 365-373.

[15] E. Guilmineau, P. Queutey, Numerical study of dynamic stall on several airfoil sections, AIAA Journal 37 (1) (1999) 128-130.

[16] I. Gursal, R. Gordnier, M. Visbal, Unsteady aerodynamics of nonslender delta wings, Progress in Aerospace Sciences 41 (2005) 515-557.

[17] J. Henkner, Phenomena of dynamic stall on swept wings, in: 22nd International Congress of the Aeronautical Sciences, ICAS Paper 2000-2.9.2, Aug.-Sep. 2000.

[18] A. Huang, C. Folk, C. Silva, B. Christensen, Y. Chen, C.M. Ho, F. Jiang, C. Grosjean, Y.C. Tai, G.B. Lee, M. Chen, S. Newbern, Application of
MEMS devices to delta wing aircraft: From concept development to transonic flight test, AIAA Paper 2001-0124, Jan. 2001.

[19] S.A. Huyer, M.C. Robinson, M.W. Luttges, Unsteady aerodynamic loading produced by a sinusoidally oscillating delta wing, Journal of Aircraft 29 (3) (1992) 366-373.

[20] S.P. LeMay, S.M. Batill, R.C. Nelson, Vortex dynamics on a pitching delta wing, Journal of Aircraft 27 (2) (1990) 131-138.

[21] J.M. Luckring, Reynolds number and leading-edge bluntness effects on a 65-deg delta wing, AIAA Paper 2002-419, Jan. 2002.

[22] P.E. Morgan, M.R. Visbal, Simulation of unsteady three-dimensional separation on a pitching wing, AIAA Paper 2001-2709, June 2001.

[23] M.R. Soltani, M.B. Bragg, J.M. Brandon, Measurements on an oscillating 70-deg delta wing in subsonic flow, Journal of Aircraft 27 (3) (1990) 211217.

[24] A. Spentzos, G. Barakos, K. Badcock, B. Richards, CFD study of 3-D dynamic stall, AIAA Paper 2005-1107, Jan. 2005.

[25] W.Z. Strang, R.F. Tomaro, M.J. Grismer, The defining methods of Cobalt 60 : A parallel, implicit, unstructured Euler/Navier-Stokes flow solver, AIAA Paper 99-786 Jan. 1999.

[26] G.S. Taylor, A. Kroker, I. Gursul, Passive flow control over flexible nonslender delta wings, AIAA Paper 2005-865, Jan. 2005.

[27] R.F. Tomaro, W.Z. Strang, L.N. Sankar, An implicit algorithm for solving time dependent flows on unstructured grids, AIAA Paper 97-333, Jan. 1997.

[28] P. Wernert, G. Koerber, F. Wietrich, M. Raffel, J. Kompenhans, Demonstration by PIV of the non-reproducibility of the flow field around an airfoil pitching under deep dynamic stall conditions and consequences thereof, Aerospace Science and Technology 2 (1997) 125-135. 